\title{
ONTOLOGY-BASED ACCESS TO TEMPORAL DATA WITH ONTOP: A FRAMEWORK PROPOSAL
}

\author{
ElEm GÜZEL KALAYCI $^{a}$, Sebastian BRANDT ${ }^{b}$, DiEgo CALVANESE $^{a}$, \\ VLADISLAV RYZHIKOV ${ }^{c}$, GUOHUI XIAO $^{a, *}$, MichaEL ZAKHARYASCHEV $^{c, d}$ \\ ${ }^{a}$ KRDB Research Centre for Knowledge and Data \\ Free University of Bozen-Bolzano, Piazza Domenicani 3, 39100 Bolzano, Italy \\ e-mail: xiaodinf.unibz.it \\ ${ }^{b}$ Siemens CT \\ Otto-Hahn-Ring 6, 81739 München, Germany \\ ${ }^{c}$ Department of Computer Science and Information Systems \\ Birkbeck, University of London, Malet St., London WC1E 7HX, UK \\ ${ }^{d}$ National Research University Higher School of Economics \\ 3 Kochnovsky Proezd, 125319, Moscow, Russia
}

\begin{abstract}
Predictive analysis gradually gains importance in industry. For instance, service engineers at Siemens diagnostic centres unveil hidden knowledge in huge amounts of historical sensor data and use it to improve the predictive systems analysing live data. Currently, the analysis is usually done using data-dependent rules that are specific to individual sensors and equipment. This dependence poses significant challenges in rule authoring, reuse, and maintenance by engineers. One solution to this problem is to employ ontology-based data access (OBDA), which provides a conceptual view of data via an ontology. However, classical OBDA systems do not support access to temporal data and reasoning over it. To address this issue, we propose a framework for temporal OBDA. In this framework, we use extended mapping languages to extract information about temporal events in the RDF format, classical ontology and rule languages to reflect static information, as well as a temporal rule language to describe events. We also propose a $S P A R Q L$-based query language for retrieving temporal information and, finally, an architecture of system implementation extending the state-of-the-art OBDA platform Ontop.
\end{abstract}

Keywords: metric temporal logic, ontology-based data access, SPARQL query, Ontop.

\section{Introduction}

Analysis of log sensor data is an important problem in industry as it reveals crucial insights into the performance and conditions of devices. The outcomes of this analysis, known as retrospective diagnostics, enable IT experts to improve the capabilities of real-time systems monitoring abnormal or potentially dangerous events developing in devices, in particular, the systems that perform predictive diagnostics. For complex devices (including those we consider in the use case below), such events do not amount to simply measurable instances (say, the

*Corresponding author temperature above $100^{\circ} \mathrm{C}$ ). Instead, they involve a number of measurements from sensors attached to a device, each having a certain temporal duration and occurring in a certain temporal sequence.

In this paper we focus on a use case by Siemens, which maintains thousands of devices related to power generation, including gas and steam turbines. It monitors these devices and provides operational support for them through a global network of more than 50 remote diagnostic centres that are linked to a common database centre. Siemens wants to employ retrospective and predictive diagnostics in order to anticipate problems with turbines and take appropriate countermeasures. A 
major challenge in this task comes from the combined need of dealing with complex temporal information and with heterogeneous data, considering that the various turbine models have different schemas of the underlying databases storing sensor measurements.

To deal with heterogeneity of data, we rely on ontology-based data access (OBDA), which was first suggested by Calvanese et al. (2007) and Poggi et al. (2008) as a means to detach the conceptual layer of classes and properties, to be exposed to end-users, from the complex structure of the underlying data sources, which thus can be hidden to users. In fact, those classes and properties are mapped to the data source schemas by means of a declarative specification. In addition, an ontology is used to model the domain of interest by asserting conceptual relations (for instance, isA) between the classes and properties. In the solution we propose here, OBDA allows us to detach the conceptual view of an event located in time-such as 'HighRotorSpeed of turbine tb01 in the period from 2017-06-06 12:20:00 to 2017-06-06 12:20:03'-from concrete databases that store the log of the sensors of that turbine.

There are several systems implementing the OBDA approach, some of which (e.g., Ontop ${ }^{1}$ and Morph ${ }^{2}$ ) are freely available, while others (e.g., Stardog 3 , Mastrd4, and Ultrawrap ${ }^{5}$ ) are distributed under commercial licences. For a recent survey of OBDA, we refer to the work by Xiao et al. (2018).

Unfortunately, none of the available OBDA systems supports access to temporal data well enough to work with the events relevant to our use case. On the one hand, the common mapping languages are not tailored towards extracting validity time intervals of conceptual assertions. On the other hand-and this is a more serious limitation-the supported ontology languages do not allow one to construct classes and properties whose temporal validity depends on the validity of other classes and properties, which is essential for defining complex temporal events. In fact, the OBDA systems used industrially are based on lightweight (non-temporal) ontology languages, such as the OWL $2 Q L$ profile of the Web Ontology Language $O W L 2$, in order to guarantee maximally efficient query answering by a reduction to standard database query evaluation. When limited to a classical ontology language, one approach to enable the extraction of temporal events is by extending the end-user query language with various temporal operators (see, e.g., Gutiérrez-Basulto and Klarman, 2012; Baader et al., 2013; Borgwardt et al., 2013; Möller et al., 2013; Klarman and Meyer, 2014; Özçep et al., 2014; Kharlamov

${ }^{1}$ http://ontop.inf.unibz.it

${ }^{2}$ https://github.com/oeg-upm/morph-rdb

3 http://stardog.com

4 http: / / www . obdasystems.com/mastro

5 https: / / capsenta.com et al., 2016). However, this approach leaves the burden of encoding the complex events in temporal queries to the end-user. In the Siemens scenario, this is a prohibitive limitation since the end-users are service engineers who are not trained in temporal knowledge representation.

Therefore, we are interested in a more expressive setting, where the ontology language is extended by temporal operators that are capable of defining complex temporal events. Extensions of lightweight ontology languages with temporal operators of linear temporal logic (LTL) such as 'next time', 'always', and 'eventually' have been suggested by Artale et al. (2013; 2015a) and Gutiérrez-Basulto et al. (2016a). However, in our use case and other similarly complex scenarios, sensors tend to send data at irregular time intervals. Moreover, even if sensor data arrives regularly, due to deadband settings the data might be stored in a data collector only when the last arrived sensor measurement is within a certain value distance from the previously transmitted one. To cope with this situation, one could replace point-based LTL with interval-based temporal logics. Thus, Artale et al. (2015b) and Kontchakov et al. (2016) proposed extensions of ontology languages and datalog programs with the Allen operators on temporal intervals used in the Halpern-Shoham logic, $\mathcal{H S}$ (Halpern and Shoham, 1991). Unfortunately, it is not possible to express in $\mathcal{H S}$ numerical constraints such as "within the next 10 minutes, main flame will continuously be on for at least 10 seconds.' A more suitable temporal representation formalism for our use case is the metric temporal logic, MTL, over a dense timeline, which was originally introduced for modeling and reasoning about real-time systems (Koymans, 1990; Alur and Henzinger, 1993). In fact, the extension datalogMTL of datalog with MTL operators proposed by Brandt et al. (2017a) appears to be both capable of capturing the events of interest for the diagnostic tasks at Siemens and suitable for the OBDA scenario. It is to be noted that MTL extensions of the expressive ontology language $\mathcal{A L C}$ over discrete time have been recently considered by Gutiérrez-Basulto et al. (2016b) and Baader et al. (2017).

In this paper, through a running example from the Siemens use case, we present a framework for temporal OBDA that employs as an ontology language to describe temporal events datalog ${ }_{n r} M T L$ the non-recursive version of datalogMTL. This framework also supports the standard OWL 2 QL language to model static knowledge (such as a configuration of modules of a turbine), and extends it with non-recursive datalog rules to describe static knowledge of a more complex structure. We outline extensions of the standard mapping language $R 2 R M L$ (Das et al., 2012) and the query language SPARQL to extract information on the validity intervals of temporal predicates. Finally, we discuss an implementation of the proposed framework in the OBDA system Ontop. 


\section{Framework for temporal OBDA}

Recall that, in classical (non-temporal) OBDA, an $O B D A$ specification is a triple $\mathcal{P}=\langle\mathcal{O}, \mathcal{M}, \mathcal{S}\rangle$, where $\mathcal{O}$ is an $O W L 2 Q L$ ontology, $\mathcal{S}$ a database schema, and $\mathcal{M}$ a set of $R 2 R M L$ mapping assertions, each associating to a class or property in $\mathcal{O}$ an $S Q L$ query over $\mathcal{S}$. An $O B D A$ instance is a pair $\langle\mathcal{P}, D\rangle$, where $D$ is a database instance satisfying schema $\mathcal{S}$. Intuitively, by applying the mapping $\mathcal{M}$ to the data instance $D$, which consists in executing the $S Q L$ queries in the mapping assertions over $D$ and populating the corresponding classes and properties using the returned values, we would obtain an RDF graph $\mathcal{M}(D)$ that reflects the content of $D$ at the ontology level. The ontology $\mathcal{O}$ complements the data with background knowledge and provides a convenient vocabulary for user queries, which are formulated in the W3C standard language SPARQL. A certain answer to such a SPARQL query $\boldsymbol{q}(\vec{x})$ over $\langle\mathcal{P}, D\rangle$ is any tuple $\vec{a}$ from $D$ for which $\boldsymbol{q}(\vec{a})$ holds in all models of $\mathcal{O}$ and $\mathcal{M}(D)$. To find the certain answers, the OBDA system rewrites the ontology-mediated query (OMQ for short) $(\mathcal{O}, \boldsymbol{q})$ into an $S Q L$ query $\boldsymbol{q}^{\prime}(\vec{x})$ over $\mathcal{S}$ that satisfies the following condition: for every data instance $D$ complying with $\mathcal{S}$ and every tuple $\vec{a}$ in it, we have that $\mathcal{O}, \mathcal{M}(\mathcal{D})=$ $\boldsymbol{q}(\vec{a})$ if and only if $\vec{a}$ is an answer to $\boldsymbol{q}^{\prime}(\vec{x})$ over $D$. Thus, answering ontology-mediated queries is reduced to standard database query evaluation. Consequently, it is in $\mathrm{AC}^{0}$ for data complexity. For more details and further references, we refer to the survey by Xiao et al. (2018).

In the remainder of this section, we present our framework for temporal OBDA by introducing temporal OBDA specifications and instances, as well as a query language for those instances based on a variant of $\tau$-SPARQL (Tappolet and Bernstein, 2009).

2.1. Temporal OBDA specification. Since we want to develop temporal OBDA by extending the standard non-temporal OBDA paradigm, we now call the OWL $2 Q L$ ontology $\mathcal{O}$ above a static ontology and $\mathcal{M}$ a set of static mapping assertions. In what follows, we will extend the static vocabulary $\Sigma_{s}$ of classes and properties occurring in $\mathcal{O}$ and $\mathcal{M}$ by a disjoint temporal vocabulary $\Sigma_{t}$. We now describe static ontologies in greater detail using an example from the Siemens use case.

2.1.1. Static ontology. At Siemens, the devices used for power generation are monitored by various types of sensors that report the temperature, pressure, vibration, rotor speed, and other relevant measurements. In order to model the static knowledge regarding the machines and their deployment profiles, sensor configurations, component hierarchies, and functional profiles, Siemens designed an OWL 2 QL ontology (Kharlamov et al., 2017), a snippet of which is given in Example 1 below using the syntax of description logics (Baader et al., 2007).

Example 1. The signature $\Sigma_{s}$ of the Siemens static ontology $\mathcal{O}$ contains the following classes (in the first three lines) and properties (in the fourth line)

Train, Turbine, GasTurbine, SteamTurbine, TurbinePart, PowerTurbine, Burner, Sensor, RotationSpeedSensor, TemperatureSensor, isMonitoredBy, isPartOf, isDeployedln.

Some of the axioms (inclusions and equivalences between classes) from $\mathcal{O}$ are shown below:

$$
\begin{aligned}
& \text { GasTurbine } \sqsubseteq \text { Turbine, } \\
& \text { SteamTurbine } \sqsubseteq \text { Turbine, } \\
& \text { RotationSpeedSensor } \sqsubseteq \text { Sensor, } \\
& \text { TemperatureSensor } \sqsubseteq \text { Sensor, } \\
& \text { PowerTurbine } \sqsubseteq \text { TurbinePart, } \\
& \text { Burner } \sqsubseteq \text { TurbinePart } \\
& \exists \text { isMonitoredBy } \sqsubseteq \text { TurbinePart, } \\
& \text { JisMonitoredBy }^{-} \sqsubseteq \text { Sensor, } \\
& \exists \text { isPartOf } \equiv \text { TurbinePart, } \\
& \text { JisPartOf }^{-} \sqsubset \text { Turbine, } \\
& \exists \text { isDeployedln } \sqsubseteq \text { Turbine, } \\
& \text { ᄏisDeployedln }{ }^{-} \sqsubseteq \text { Train. }
\end{aligned}
$$

For a property $P$, the expression $\exists P$ denotes the domain of $P$, while $\exists P^{-}$denotes the range of $P$. Thus, the last two axioms say that the domain of the property isDeployedln is Turbine and the range is Train.

Unfortunately, OWL $2 Q L$ has a limited expressive power and is not able to capture all the static knowledge that is relevant to the Siemens use case. In particular, it does not allow predicates of arity greater than 2 and intersection on the left-hand side of inclusions. A well-known language with these missing constructs is standard datalog (Abiteboul et al., 1995). Note, however, that answering datalog queries is P-complete for data complexity, and so it cannot be reduced to database query evaluation in general. A typical example of a datalog rule from our ontology is given in the next section.

2.1.2. Static rules. In the Siemens use case, some turbine parts are monitored by a number of different sensors, say, a temperature sensor and a rotation speed sensor. This situation can be readily described by a datalog rule with a ternary predicate in the head and a complex body, such as the one in the example below, but not by $O W L 2 Q L$ axioms.

\footnotetext{
${ }^{6}$ In description logic parlance, classes are called concepts and correspond to unary predicates, while properties are called roles and correspond to binary predicates.
} 
Example 2. The datalog rule

ColocTempRotSensors $(t b, t s, r s) \leftarrow$ Turbine $(t b)$, isPartOf $(b r, t b)$, Burner $(b r)$, isMonitoredBy $(b r, t s)$, TemperatureSensor $(t s)$, isPartOf $(p t, t b)$, PowerTurbine $(p t)$, isMonitoredBy $(p t, r s)$, RotationSpeedSensor $(r s)$

is supposed to say that a temperature sensor $t s$ and a rotation speed sensor $r s$ are co-located in the same turbine $t b$ if $t b$ has a part $b r$, which is a burner, monitored by $t s$, and has another part $p t$, which is a power turbine, monitored by rs.

We denote by $\mathcal{R}$ a set of static datalog rules and by $\Sigma_{s}$ the static signature that contains the symbols from both $\mathcal{O}$ and $\mathcal{R}$. Note that the rules in $\mathcal{R}$ may contain classes and properties from $\mathcal{O}$. In order to make sure that answering queries mediated by $\mathcal{O} \cup \mathcal{R}$ is reducible to database query evaluation, we impose two restrictions on $\mathcal{R}$ : $(i)$ it has to be non-recursive and ( $i$ ) the predicates in the head of rules in $\mathcal{R}$ cannot occur in $\mathcal{O}$.

The static ontology language considered so far is supposed to represent time-independent knowledge and falls short of capturing temporal events that are required in the Siemens use case.

2.1.3. Temporal rules. Siemens is interested in detecting abnormal situations in the working equipment as well as in monitoring running tasks in order to see whether they proceed ordinarily. A typical event that is crucial to monitor is a normal start of a turbine. This event is rather complex and composed of various subevents that are distributed over time and characterized by a temporal duration. One of these subevents, Purging Is Over, is described in the example below.

Example 3. Purging Is Over is a complex temporal event for a given turbine $t b$, which is characterized by the following:

(i) there is a pair of sensors co-located in the turbine $t b$, one of which is a rotor speed sensor $r s$ and the other one a temperature sensor $t s$;

(ii) the temperature sensor $t s$ detects that the main flame was burning for at least 10 seconds;

(iii) at the same time, the following happened within the preceding 10 minutes:

- the rotor speed sensor $r s$ measured a speed of at most $1000 \mathrm{rpm}$ for at least 30 seconds, and

- within the following 2 minutes, the rotor speed sensor rs measured a speed of at least 1260 rpm for at least 30 seconds.

We illustrate the described event in Fig. 10.

Here, we assume that the horizontal axis represents time and PurginglsOver $(t b)$ holds at a moment of time if prior to that moment MainFlameOn $(t s)$,

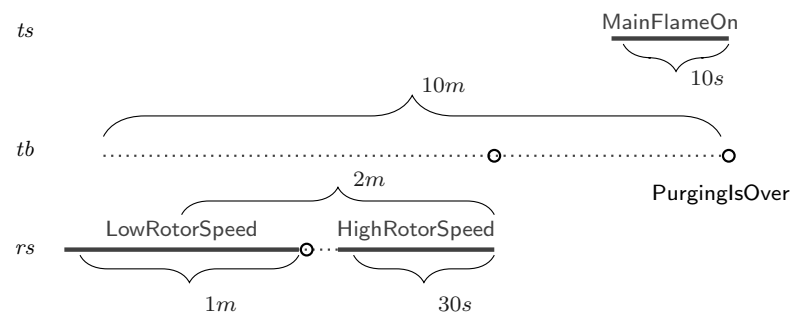

Fig. 1. Event of Example 3.

LowRotorSpeed $(r s)$, and HighRotorSpeed $(r s)$ had occurred following the depicted pattern.

In our examples, we shall also use the temporal event Main Flame Is On, which happens for a given temperature sensor $t s$ when the main flame had been above the threshold (of 1.0) for 10 seconds continuously in the past.

Temporal diagnostic patterns of this sort can be described by means of a datalog $\log _{n r} M T L$ program (Brandt et al., 2017a), which is a set of non-recursive datalog rules extended with temporal operators of the metric temporal logic MTL under the continuous semantics over the real numbers $(\mathbb{R},<)$ (Alur and Henzinger, 1993). In such programs, we require a (countably infinite) list of temporal predicates (with the corresponding arities) that is disjoint from the list of static predicates. Intuitively, each temporal predicate may be true on some domain objects at certain moments of time and false on other domain objects and time instants. Under this semantics, static predicates are assumed to be time-independent, that is, to hold true or false on a given tuple of domain objects at all times. The event Purging Is Over can be formalized by the following datalog $\log _{n r}$ MTL program $\mathcal{T}$.

Example 4. The program $\mathcal{T}$ consists of five rules:

$$
\begin{aligned}
& \text { PurginglsOver }(t b) \leftarrow \text { MainFlameOn }(t s), \\
& \vartheta_{(0,10 m]}\left(\Xi_{(0,30 s]} \text { HighRotorSpeed }(r s),\right. \\
& \left.\vartheta_{(0,2 m]} \boxminus_{(0,1 m]} \text { LowRotorSpeed }(r s)\right), \\
& \text { ColocTempRotSensors }(t b, t s, r s), \\
& \text { MainFlameOn }(t s) \leftarrow \boxminus_{[0 s, 10 s]} \text { MainFlameUpTH }(t s), \\
& \text { MainFlameUpTH }(t s) \leftarrow \operatorname{mainFlame~}(t s, v), v \geq 1.0, \\
& \text { HighRotorSpeed }(r s) \leftarrow \operatorname{rotorSpeed~}(r s, v), v>1260, \\
& \text { LowRotorSpeed }(r s) \leftarrow \operatorname{rotorSpeed}(r s, v), v<1000 .
\end{aligned}
$$

Here, ColocTempRotSensors is the static (time independent) predicate from Example 2, The unary numerical built-in predicates $v \geq 1.0, v>1260$, and $v<1000$ are also static. The predicates rotorSpeed, HighRotorSpeed, LowRotorSpeed, and MainFlameUpTH are temporal; $\operatorname{rotorSpeed}(r s, v)$ holds true at a time instant $t$ if and only if $v$ is the rotor speed 
measured by the sensor $r s$ at $t$; HighRotorSpeed $(r s)$ holds true exactly at those time instants $t$ where rotorSpeed $(r s, v)$ holds for some value $v>1260$; and similarly for LowRotorSpeed and MainFlameUpTH. The temporal predicate MainFlameOn is defined using the MTL operator $\Xi_{[0 s, 10 s]}$ : namely, MainFlameOn $(t s)$ holds true at time instant $t$ if MainFlameUpTH $(t s)$ holds at all time instants $t^{\prime} \in[t-10 s, t]$. Finally, PurginglsOver is a temporal predicate that, in addition to the $\boxminus$ operator, uses the MTL operator $\theta$. For example, $\vartheta_{(0,10 m]}$ is interpreted as follows: $\nabla_{(0,10 m]} \varphi$ holds true at $t$ if and only if $\varphi$ holds true at some time instant $t^{\prime} \in[t-10 \mathrm{~m}, t)$.

We denote by $\Sigma_{t}$ the set of temporal predicates from $\mathcal{T}$ and note that, in datalog ${ }_{n r} M T L$ programs like $\mathcal{T}$, only predicates from $\Sigma_{t}$ can occur in the head of the rules, whereas their bodies can contain predicates from both $\Sigma_{t}$ and $\Sigma_{s}$. (Thus, intuitively, the temporal rules in $\mathcal{T}$ define temporal predicates in terms of both temporal and static ones.) In the example above, $\Sigma_{t}$ comprises the predicates

mainFlame, rotorSpeed,

PurginglsOver, MainFlameOn, MainFlameUpTH,

HighRotorSpeed, LowRotorSpeed.

We emphasise once again that data $\log _{n r} M T L$ programs are required to be non-recursive. Without such a restriction, the data complexity of answering ontology-mediated queries in our framework becomes P-hard, which makes a reduction to database query evaluation impossible. For non-recursive datalog $\log _{n r} M T L$ rules, the $\mathrm{AC}^{0}$ data complexity follows from the work by Brandt et al. (2017a), if we restrict the OWL $2 Q L$ ontologies to the DL-Lite rdfs $_{s}$ fragment (Calvanese et al., 2007). Currently, we are working on extending this result to full $O W L 2 Q L$ in a way similar to the extension of $O W L 2 Q L$ with the temporal operators of linear temporal logic LTL (Artale et al., 2015a).

2.1.4. Databases and mappings. In our approach, we assume that databases have generic schemas. However, since in temporal OBDA, we have to deal with temporal data, we are particularly interested in databases with tables containing timestamp columns.

Example 5. An example data schema $\mathcal{S}$ for the Siemens data, including sensor measurements and deployment details, can look as follows (the primary keys of the tables are underlined):

tb_measurement (tstmp, s_id, value), tb_sensors( $\underline{\text { s_id }}$, s_type, mnted_part, mnted_tb), tb_deployment (turbine_id, turbine_type, deployed_in), tb_components (turbine_id, comp_id, comp_type).
Table 1. Example Siemens use case tables

\begin{tabular}{|c|c|c|}
\hline \multicolumn{3}{|c|}{ tb_measurement } \\
\hline tstmp & s_id & value \\
\hline \hline 17-06-06 12:20:00 & rs01 & 570 \\
17-06-06 12:21:00 & rs01 & 680 \\
17-06-06 12:21:30 & rs01 & 920 \\
17-06-06 12:22:50 & rs01 & 1278 \\
17-06-06 12:23:40 & rs01 & 1310 \\
& $\ldots$ & \\
17-06-06 12:32:30 & mf01 & 2.3 \\
17-06-06 12:32:37 & mf01 & 1.8 \\
17-06-06 12:32:45 & mf01 & 0.9 \\
& $\ldots$ & \\
\hline
\end{tabular}

\begin{tabular}{|c|c|c|c|}
\hline \multicolumn{4}{|c|}{ tb_sensors } \\
\hline s_id & s_type & mnted_part & mnted_tb \\
\hline \hline rs01 & 0 & pt01 & tb01 \\
mf01 & 1 & b01 & tb01 \\
& $\ldots$ & $\ldots$ & \\
\hline
\end{tabular}

\begin{tabular}{|c|c|c|}
\hline \multicolumn{3}{|c|}{ tb_components } \\
\hline turbine_id & comp_id & comp_type \\
\hline \hline tb01 & pt01 & 0 \\
tb01 & b01 & 1 \\
& $\cdots$ & \\
\hline
\end{tabular}

Three snippets of data from the Siemens use case tables tb_measurement, tb_sensor, and tb_components, are given in Table 1 .

In classical OBDA, mapping assertions take the form $\varphi(\vec{x}) \rightsquigarrow \psi(\vec{x})$, where $\varphi(\vec{x})$ is a query over the schema $\mathcal{S}$ and $\psi(\vec{x})$ is an atom with a predicate from $\Sigma_{s}$ and variables $\vec{x}$ (Xiao et al., 2018).

Example 6. Given the static ontology $\mathcal{O}$ and the signature $\Sigma_{s}$ from Example 1 as well as the schema $\mathcal{S}$ from Example 5, the following are examples of mapping assertions:

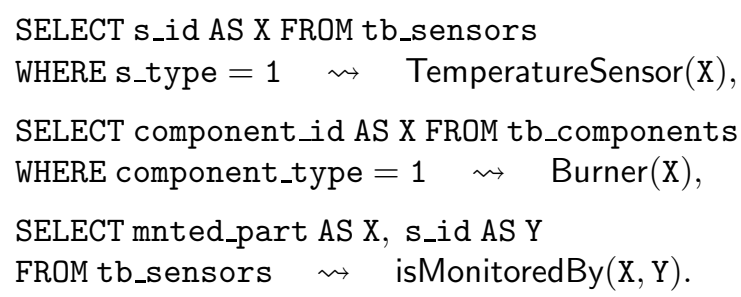

To explain how the mapping assertions work, consider the database from Example 5 and the SQL query on the left-hand side of the first assertion. The result of executing this query over the database is a table with a single column named $\mathrm{X}$ and containing a tuple $\mathrm{mf} 01$. The right-hand side of the mapping indicates that, according to the database, the fact TemperatureSensor(mf01) holds true (as well as other such facts if the query returns more answers). 
By applying these mapping assertions to the database from Example 5, we extract the following facts (ground atoms):

$$
\begin{aligned}
& \text { Burner(b01), TemperatureSensor(mf 01), } \\
& \text { isMonitoredBy(pt01, rs01), } \\
& \text { isMonitoredBy(b01, mf 01). }
\end{aligned}
$$

We call the mapping assertions that extract ground atoms for predicates from $\Sigma_{s}$ static, and use $\mathcal{M}_{s}$ to denote their sets.

On the other hand, to deal with temporal predicates from $\Sigma_{t}$, we also require temporal mapping assertions of the form

$$
\varphi(\vec{x}, \text { begin, end }) \rightsquigarrow \psi(\vec{x}) @\left\langle t_{\text {begin }}, t_{\text {end }}\right\rangle,
$$

where $\varphi(\vec{x}$, begin, end $)$ is a query over $\mathcal{S}$ such that the variables begin and end are mapped to values of the date/time format, $\psi$ is a predicate from $\Sigma_{t}, t_{\text {begin }}$ is either the variable begin or a constant temporal value (timestamp) including $\infty,-\infty$ (and similarly for $t_{\text {end }}$ ), '〈' is either '(' or [', and ' $>$ ' is either ')' or ]'. For example, $\psi(\vec{x}) @[$ begin, $\infty)$ means that $\psi(\vec{x})$ holds at every time instant in the interval [begin, $\infty$ ), and the variables $\vec{x}$ and begin are instantiated by the query on the left-hand side of the mapping assertion. Temporal mapping assertions are required to define predicates from $\Sigma_{t}$ only. We denote by $\mathcal{M}_{t}$ sets of such mapping assertions.

Example 7. Given $\Sigma_{t}$ and $\mathcal{T}$ from Example 4 and the schema $\mathcal{S}$ from Example 5, the following is an example of a temporal mapping assertion:

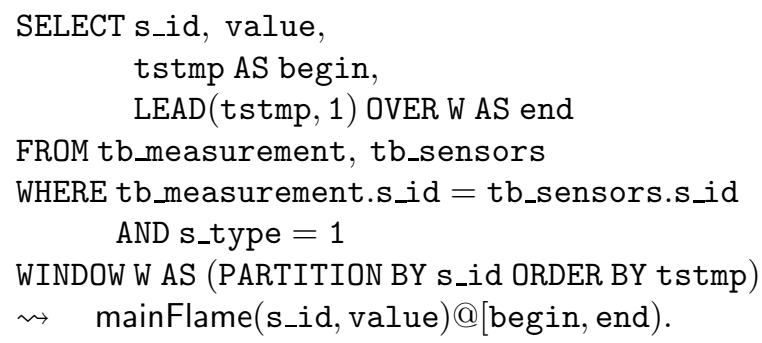

This mapping assertion extracts from the database in Example 5 the following temporal facts:

mainFlame(mf01, 2.3)@[12:32:30, 12:32:37),

mainFlame(mf01, 1.8)@[12:32:37, 12:32:45).

For instance, the first of them states that the main flame sensor $\mathrm{mf} 01$ was registering the value 2.3 in the interval [12:32:30, 12:32:37). Note that the interval is left-closed and right-open, which reflects the logic of how turbine sensor outputs are produced: namely, a sensor outputs a value when the result of a measurement differs from the previously returned value by a fixed threshold. Similarly, by means of an appropriate mapping, we shall extract the temporal fact stating that the rotation sensor $\mathrm{rs} 01$ was registering the speed 570 in the interval [12:20:00, 12:21:00).
Table 2. Languages of the components in temporal OBDA.

\begin{tabular}{|c|c|c|c|}
\hline component & $\begin{array}{c}\text { defines } \\
\text { predicates in }\end{array}$ & $\begin{array}{c}\text { in terms of } \\
\text { predicates in }\end{array}$ & language \\
\hline \hline $\mathcal{M}_{s}$ & $\Sigma_{s}$ & $\mathcal{S}$ & $R 2 R M L$ \\
$\mathcal{M}_{t}$ & $\Sigma_{t}$ & $\mathcal{S}$ & $R 2 R M L$ \\
$\mathcal{O}$ & $\Sigma_{s}$ & $\Sigma_{s}$ & OWL 2QL \\
$\mathcal{R}$ & $\Sigma_{s}$ & $\Sigma_{s}$ & non-recursive \\
& & & datalog \\
$\mathcal{T}$ & $\Sigma_{t}$ & $\Sigma_{s} \cup \Sigma_{t}$ & datalog ${ }_{n r} M T L$ \\
\hline
\end{tabular}

2.1.5. Temporal OBDA specification and instance. An OBDA specification in the temporal OBDA framework is the tuple

$$
\mathfrak{S}=\left\langle\Sigma_{s}, \Sigma_{t}, \mathcal{M}_{s}, \mathcal{M}_{t}, \mathcal{O}, \mathcal{R}, \mathcal{T}, \mathcal{S}\right\rangle
$$

where $\Sigma_{s}$ is a static signature, $\Sigma_{t}$ a temporal signature, $\mathcal{M}_{s}$ a set of static and $\mathcal{M}_{t}$ a set of temporal mapping assertions, $\mathcal{O}$ is a standard OBDA ontology in $\Sigma_{s}, \mathcal{R}$ a set of static rules in $\Sigma_{s}, \mathcal{T}$ a set of temporal rules in $\Sigma_{t}$, and $\mathcal{S}$ a database schema. In Table 2, we clarify the intuition behind the different components of $\mathfrak{S}$ and the associated specification languages. A temporal OBDA instance $\mathfrak{I}$ is a pair $\langle\mathfrak{S}, D\rangle$, where $\mathfrak{S}$ is a temporal OBDA specification and $D$ a database instance compliant with the database schema $\mathcal{S}$ in $\mathfrak{S}$. We next discuss languages for querying temporal OBDA instances.

2.2. Ontology-mediated query answering. A popular query language in standard (atemporal) OBDA is the language of conjunctive queries (Calvanese et al., 2007). In our temporal setting, a conjunctive query is a first-order formula of the form

$$
\boldsymbol{q}(\vec{x}, \vec{\iota})=\exists \vec{y}, \vec{\tau} \Phi(\vec{x}, \vec{y}, \vec{\iota}, \vec{\tau})
$$

where $\Phi$ is a conjunction of atoms of the form $P(\vec{z})$ with $P$ from $\Sigma_{s}$, atoms of the form $Q(\vec{z}) @ \varrho$ with $Q$ from $\Sigma_{t}, \vec{z} \subseteq \vec{x} \cup \vec{y}$ and $\varrho \in \vec{\imath} \cup \vec{\tau}$, and possibly built-in numerical predicates over the timeline. Here, $\varrho$ is a variable over temporal intervals (and $\vec{\imath}, \vec{\tau}$ are lists of such variables). A certain answer to $\boldsymbol{q}(\vec{x}, \vec{\iota})$ over a temporal OBDA instance $\mathfrak{I}$ is a tuple $\vec{a}$ of constants from $D$ and a tuple $\vec{\alpha}$ of temporal intervals (of the form defined above, say, [12:20:00, 15:00:00) or [12:20:00, $\infty)$ ) such that $\boldsymbol{q}(\vec{a}, \vec{\alpha})$ holds true in every temporal model of the ontology $\mathcal{O} \cup \mathcal{R} \cup \mathcal{T}$ and the sets of ground atoms extracted from $D$ by $\mathcal{M}_{s}$ and $\mathcal{M}_{t}$. One could also require that the intervals in $\vec{\alpha}$ only use those time instants that are explicitly mentioned in $D$. A more expressive query language can be obtained by extending conjunctive queries with Allen's interval relations such as ' $\iota$ is after $\tau$ ' (Allen, 1983). 
Example 8. The conjunctive query

$$
\boldsymbol{q}_{1}(\varrho)=\text { MainFlameOn }(x) @ \varrho
$$

can be used to find the periods of time when the main flame was on for some sensor, and the conjunctive query

$$
\begin{aligned}
\boldsymbol{q}_{2}(x, \varrho)= & \text { GasTurbine }(x) \wedge \text { isDeployed } \ln (x, \operatorname{tr} 05) \wedge \\
& \text { PurginglsOver }(x) @ \varrho
\end{aligned}
$$

can be used to find the gas turbines that were deployed in the train with the ID tr05 and the time periods of their accomplished purgings. Observe that, for the temporal facts shown in Example 7, the program $\mathcal{T}$ from Example 4 and $\mathcal{R}, \mathcal{O}$ as above, the certain answer to the query $\boldsymbol{q}_{1}$ will be the interval [12:32:40,12:32:45). This is because MainFlameUpTH(mf01)@[12:32:30, 12:32:37) and MainFlameUpTH(mf01)@[12:32:37, 12:32:45) (and so also MainFlameUpTH(mf01)@[12:32:30, 12:32:45)) hold true in every temporal model of the ontology $\mathcal{O} \cup \mathcal{R} \cup \mathcal{T}$ and the sets of ground atoms extracted from the data instance $D$ by means of the mappings $\mathcal{M}_{s}$ and $\mathcal{M}_{t}$, and so the same holds also for MainFlameOn(mf01)@[12:32:40, 12:32:45).

Answering conjunctive queries in temporal OBDA turns out to be a difficult problem, in both theory and practice; see, e.g., the work by Artale et al. (2013; 2015a), who considered extensions of OWL 2 QL and conjunctive queries with the operators of linear temporal logic LTL. When datalog (instead of OWL $2 Q L$ ) is used as a static ontology language, answering temporal conjunctive queries becomes easier (in terms of query rewriting algorithms); we refer to Section 5 for practical applications and further discussion.

2.2.1. Temporal SPARQL. In the temporal OBDA framework we suggest in this paper, our aim is to employ as the query answering engine the OBDA platform Ontop that was designed for classical OBDA with OWL $2 Q L$ (Rodriguez-Muro et al., 2013; Calvanese et al., 2017). In the non-temporal setting, Ontop essentially supports answering SPARQL queries under the OWL $2 Q L$ direct semantics entailment regime over virtual RDF graphs populated by $R 2 R M L$ mappings and data instances stored in relational databases (Kontchakov et al., 2014).

In the temporal setting, to be compatible with the available functionalities of Ontop, we suggest a query language that is an extension of SPARQL similar to $\tau$-SPARQL proposed by Tappolet and Bernstein (2009). We remind the reader that variables in SPARQL are prefixed by '?' and that atoms take the form $? x$ a : GasTurbine (which stands for GasTurbine $(x)$ ) and $? x$ : isDeployedln ? $y$ (which stands for isDeployedln $(x, y)$ ). (Relations of arity higher than 2 , such as ColocTempRotSensors mentioned above, are not supported directly in our language and have to be handled via reification; see, e.g., the work by Calvanese and De Giacomo (2003) and the references therein. Atoms like these are used for the static predicates from $\Sigma_{s}$ (such as GasTurbine, isDeployedln). Temporal predicates from $\Sigma_{t}$ (such as PurginglsOver) have to be followed by a suffix $@\left\langle ? e_{1}, ? v_{1}, ? v_{2}, ? e_{2}\right\rangle$, where $? e_{1}$ is a Boolean variable evaluating to either 'true' or 'false', depending on whether the interval where the predicate holds is left-closed or left-open (and similarly for $? e_{2}$ indicating right-closedness or right-openness), while $? v_{1}$ and $? v_{2}$ are variables over date/time whose values respectively indicate from when and until when the predicate holds.

Example 9. The conjunctive query $\boldsymbol{q}_{1}$ from Example 8 can be represented as the following temporal SPARQL query:

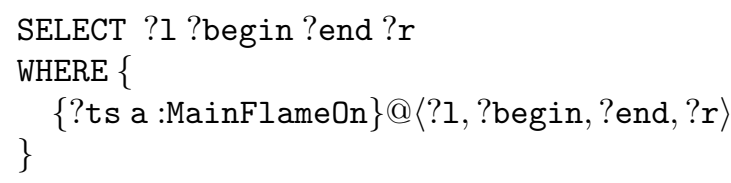

whereas the conjunctive query $\boldsymbol{q}_{2}$ is represented as

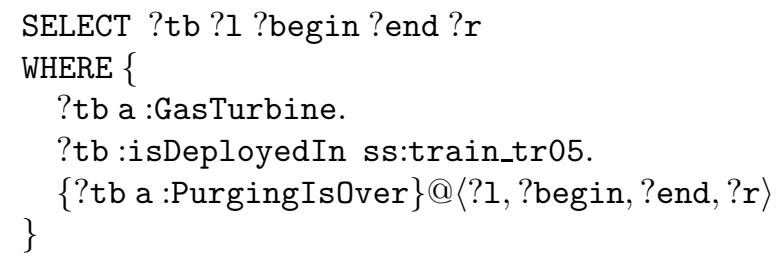

As explained above, the certain answer to the query $\boldsymbol{q}_{1}$ with ontology $\mathcal{O} \cup \mathcal{R} \cup \mathcal{T}$, data instance $D$, as well as mappings $\mathcal{M}_{s}$ and $\mathcal{M}_{t}$, contains the tuple (true, 12:32:40, 12:32:45, false).

2.2.2. On temporal RDF graphs. There does not seem to exist a standardized way of representing temporal data as RDF graphs; we refer to a few relevant proposals by Gutiérrez et al. (2005), Tappolet and Bernstein (2009), and Grandi (2010). Although our temporal OBDA framework does not presuppose any materialization of relational data in the form of an RDF graph (which will be discussed in Section 3), we advocate the use of RDF dataset:7 comprising a distinguished graph and a set of named RDF graphs, following the model of RDF stream proposed by the W3C RDF Stream Processing Community Group.

More specifically, to model temporal facts, for each relevant temporal interval we introduce a graph identifier and collect the triples that hold within this interval into the respective graph. The details of the interval (namely,

7 https://www.w3.org/TR/rdf11-datasets/

\&ttp://streamreasoning.github.io/RSP-QL/ RSP_Requirements_Design_Document/ 
the beginning and the end) of the graph identifier are described in the distinguished graph using the vocabulary from the W3C TIME ontology (Cox and Little, 2017). The static triples also reside in the distinguished graph.

Example 10. The temporal fact mainFlame(mf01, 2.3)@[12:32:30, 12:32:37)

from Example 7 can be represented as the named graph

$$
\text { GRAPH } g_{0}\{(\text { mf01, mainFlame, 2.3) }\}
$$

and the distinguished graph containing the following triples 9 :

( $g_{0}$, time:hasTime, $\left.i_{0}\right)$,

$\left(i_{0}\right.$, a, time:Interval $)$,

( $i_{0}$, time:isBeginninglnclusive, true),

$\left(i_{0}\right.$, time:isEndlnclusive, false),

$\left(i_{0}\right.$, time:hasBeginning, $\left.b_{0}\right)$,

( $b_{0}$, time:inXSDDateTimeStamp, '2017-06-06 12:32:30'),

$\left(i_{0}\right.$, time: hasEnd, $\left.e_{0}\right)$,

( $e_{0}$, time:inXSDDateTimeStamp, '2017-06-06 12:32:37').

In the same way, the temporal fact

$$
\text { mainFlame(mf 01, 2.3)@[12:32:37, 12:32:45) }
$$

can be modelled by a named graph $g_{1}$ and an interval $i_{1}$. Using the third rule of the program $\mathcal{T}$ from Example 4 , we obtain the fact represented by the named graph

$$
\text { GRAPH } g_{2}\{(\mathrm{mf01}, \mathrm{a}, \mathrm{MainFlameUpTH})\}
$$

and the distinguished graph

$\left(g_{2}\right.$, time:hasTime, $\left.i_{2}\right)$,

( $i_{2}$, a, time:Interval),

$\left(i_{2}\right.$, time:isBeginninglnclusive, true),

( $i_{2}$, time:isEndlnclusive, false),

$\left(i_{2}\right.$, time:hasBeginning, $\left.b_{2}\right)$,

( $b_{2}$, time:inXSDDateTimeStamp, '2017-06-06 12:32:30’),

$\left(i_{2}\right.$, time:hasEnd, $\left.e_{2}\right)$,

( $e_{2}$, time:inXSDDateTimeStamp, '2017-06-06 12:32:45').

Further, the second rule in $\mathcal{T}$ gives us the named graph

$$
\text { GRAPH } g_{3}\{(\text { mf01, a, MainFlameOn })\}
$$

and the distinguished graph

$\left(g_{3}\right.$, time:hasTime, $\left.i_{3}\right)$,

( $i_{3}$, a, time:Interval),

$\left(i_{3}\right.$, time:isBeginninglnclusive, true),

( $i_{3}$, time:isEndlnclusive, false),

$\left(i_{3}\right.$, time:hasBeginning, $\left.b_{3}\right)$,

( $b_{3}$, time:inXSDDateTimeStamp, '2017-06-06 12:32:40’),

$\left(i_{2}\right.$, time:hasEnd, $\left.e_{3}\right)$,

( $e_{3}$, time:inXSDDateTimeStamp, '2017-06-06 12:32:45').

\footnotetext{
${ }^{9}$ Note that we extended the Time ontology with the isBeginInclusive and isEndInclusive data properties, which are not currently supported.
}

In order to match this data, our query $\boldsymbol{q}_{1}$ from Example 9 has to be rewritten into the following SPARQL query with named graph variables:

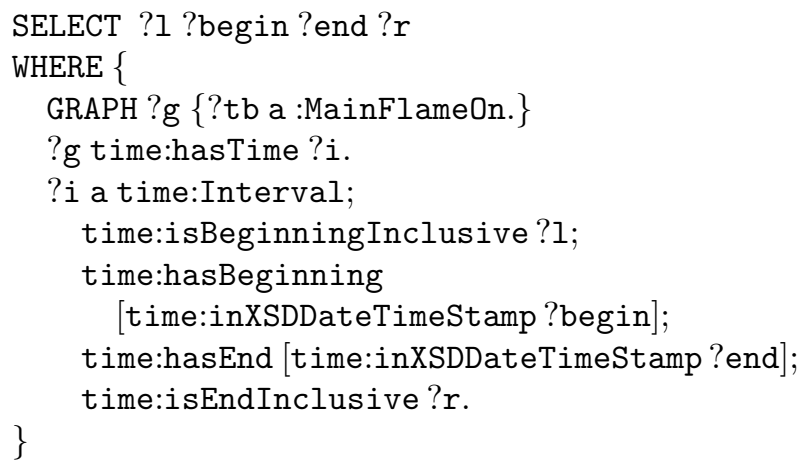

This query returns the certain answer

(true, 12:32:40, 12:32:45, false).

In fact, this rewriting is similar to the one proposed by Tappolet and Bernstein (2009).

\section{System architecture and implementation in Ontop}

In this section, we propose a system architecture of temporal OBDA by extending the OBDA platform Ontop. We first briefly describe the workflow of Ontop in the case of classical OBDA, and then discuss how to extend it to temporal OBDA.

3.1. Classical OBDA with Ontop. Ontop is a state-of-the-art OBDA system developed at the Free University of Bozen-Bolzano (Calvanese et al., 2017). Ontop supports the standard $\mathrm{W} 3 \mathrm{C}$ recommendations related to OBDA (such as OWL 2QL, R2RML, $S P A R Q L$, and the OWL $2 Q L$ entailment regime in $S P A R Q L)$. The system is available as a Protégé plugin, and an extensible open-source Java library supporting OWL API and RDF4J.

The core of an OBDA system is the query answering algorithm. Ontop uses an optimized query rewriting algorithm (Rodriguez-Muro et al., 2013) whose workflow is outlined as Algorithm 1. The algorithm takes as inputs an OBDA instance $\langle\mathcal{P}, D\rangle$ with $\mathcal{P}=\left\langle\mathcal{O}, \mathcal{M}_{s}, \mathcal{S}\right\rangle$ and a $S P A R Q L$ query $\boldsymbol{q}$, and returns the certain answers to $\boldsymbol{q}$ over $\langle\mathcal{P}, D\rangle$. The workflow can be divided into the offline and online stages. During start-up (the offline stage), Ontop $(a)$ classifies the static ontology $\mathcal{O}$ and $(b)$ compiles the classified ontology into the input mapping $\mathcal{M}_{s}$, thus obtaining the saturated mapping $\mathcal{M}_{s}^{\mathcal{O}}$ known as the T-mapping (Rodriguez-Muro et al., 2013).

During query execution (the online stage), Ontop transforms an input SPARQL query $q$ into an optimized $S Q L$ query $\boldsymbol{q}_{\text {opt }}^{\mathcal{M}, \mathcal{O}}$ exploiting the T-mapping $\mathcal{M}_{s}^{\mathcal{O}}$ and 
the database integrity constraints $\mathcal{S}$, and evaluates the generated $S Q L$ query over the database instance $D$.

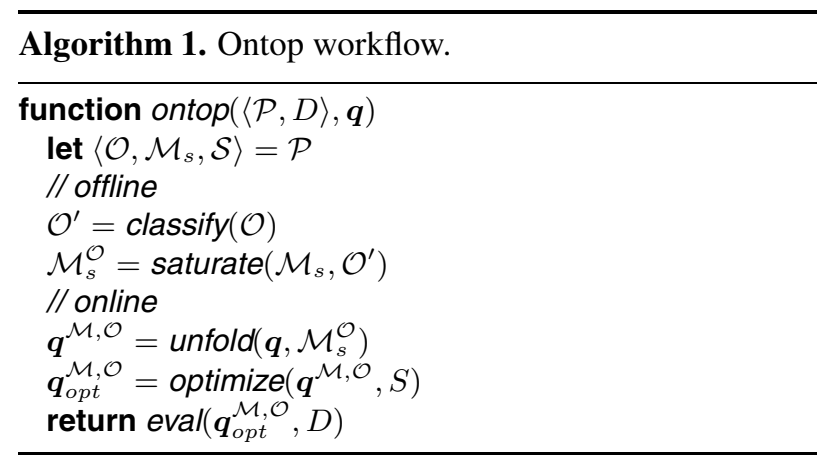

3.2. Ontop-temporal. Now we present our proposal for the temporal extension of Ontop, which we call Ontoptemporal. Specifically, we discuss the choice of concrete languages for the additional input components and how to adapt the query rewriting algorithm.

3.2.1. Concrete languages. Our principle when choosing concrete languages for the inputs is to be compliant with the relevant existing standards whenever possible; we only extend and create new syntax/languages when it is absolutely necessary.

In Ontop-temporal, we continue to use OWL $2 Q L$ for static ontologies and also allow the use of non-recursive datalog rules satisfying (i) and (ii) in Section 2.1.2 For temporal rules, there are no standard languages. The proposed concrete syntax for datalog $\log _{n r} M T L$ is inspired by datalog, $S W R L$, and SPARQL.

We continue to use $R 2 R M L$ as a mapping language. Intuitively, an $R 2 R M L$ mapping produces named graphs (see Section 10) to represent temporal information. Named graphs are supported through the R2RML construct rr:GraphMap. Alternatively, considering that it is rather verbose to map all the temporal information in $R 2 R M L$, we also extend the Ontop mapping language (Calvanese et al., 2017) to provide an alternative compact syntax close to the one used in Example 7.

As for the query language, we support both a $\tau$-SPARQL-based language and plain SPARQL as discussed in Examples 9 and 10 . Internally, the $\tau$-SPARQL-based language is treated as syntactic sugar and handled by compiling it into the corresponding plain $S P A R Q L$ query language.

3.2.2. Query answering algorithm. The algorithm of Ontop-temporal, outlined as Algorithm 2, takes as inputs a temporal OBDA instance $\langle\mathfrak{S}, D\rangle$ and a $\tau$-SPARQL or $S P A R Q L$ query $\boldsymbol{q}$, and returns the answers of $\boldsymbol{q}$ over $\langle\mathfrak{S}, D\rangle$.
Similarly to Ontop, the workflow of Ontop-temporal also consists of an offline stage, compiling $\mathfrak{S}$ into a set of mapping assertions $\mathcal{M}^{\mathfrak{S}}$, and an online stage, evaluating $\boldsymbol{q}$ over $D$ by query rewriting. The offline stage has two more steps to process the temporal components in $\mathfrak{S}$ :

(a) it saturates $\mathcal{M}_{s}^{\mathcal{O}}$ with static rules $\mathcal{R}$ as proposed by Xiao et al. (2014) and obtains the mapping $\mathcal{M}_{s}^{\mathcal{O}, \mathcal{R}}$;

(b) it saturates $\mathcal{M}_{t}$ and $\mathcal{M}_{s}^{\mathcal{O}, \mathcal{R}}$ with $\mathcal{T}$ using the algorithm by Brandt et al. (2017a; 2018), and obtains the final saturated mapping $\mathcal{M}^{\mathfrak{S}}$. In a nutshell, the algorithm computes a view for each predicate in a bottom-up fashion. The view definitions exploit $S Q L$ functions simulating the temporal operators in MTL and often result in complex $S Q L$ queries.

The online stage first converts the input query into $S P A R Q L$ when it is expressed in $\tau$-SPARQL. The optimization step also needs to be extended to handle temporal-specific constructs in SQL queries. We now present the $S Q L$ queries that we expect to be generated for the running example.

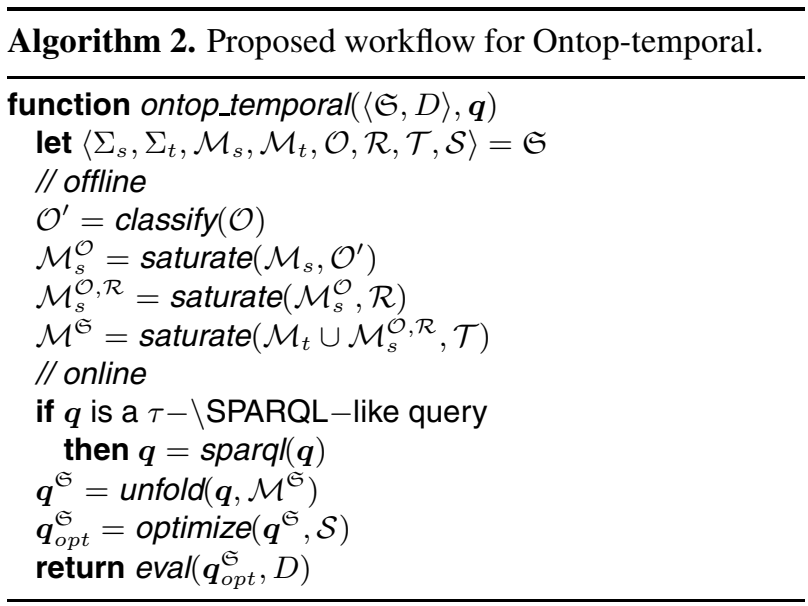

Example 11. The expected $S Q L$ translation of the temporal $S P A R Q L$ query $\boldsymbol{q}_{1}$ from Example 9 is as follows:

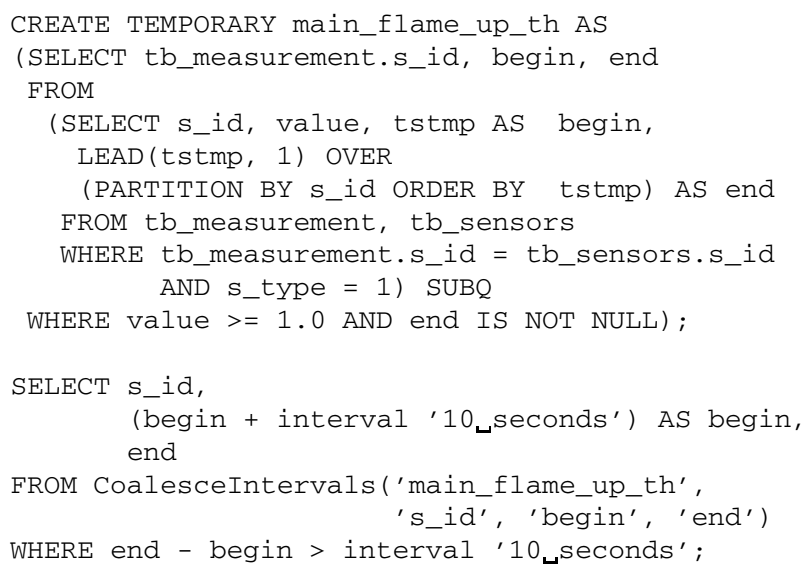


The utility function/query CoalesceIntervals is defined in Appendix. This query takes a table (main_flame_up_th in this example) with a main column (s_id) and two columns (begin and end) representing the ends of the validity interval of an object in the main column. The returned table only contains the maximal validity intervals merging (coalescing) the overlapping intervals (see how the query answer is computed in Example 8). Note that the implementation of CoalesceIntervals in this example assumes that all intervals are of the form $[x, y)$.

In more complex queries (such as $\boldsymbol{q}_{2}$ from Example [9], we use an SQL query/function TemporalJoin that, given two tables $T_{1}$ and $T_{2}$ with tuples of the form (object_id, begin, end), returns a table with the intersection of the validity intervals for each object_id (see Appendix).

\section{Experimental evaluation}

In order to show the feasibility of our approach, in this section, we present an experiment based on the running example of this paper. In the experiment, we manually computed the $S Q L$ queries produced by applying the datalog ${ }_{n r}$ MTL rewriting algorithm (Brandt et al., 2017a) and we evaluate these queries over Siemens turbine data.

The initial data provided by Siemens is a sample for one running turbine over 4 days. We replicated this sample to imitate the data for one turbine over 10 different periods ranging from 32 to 319 months. We ran the experiments on an HP Proliant server with 2 Intel Xeon X5690 processors (each with 12 logical cores at $3.47 \mathrm{GHz}$ ), $106 \mathrm{~GB}$ of RAM and five 1 TB $15 \mathrm{~K}$ RPM hard disks. The data are stored on a PostgreSQL 9.6 database server.

We evaluated four queries LowRotorSpeed $(x) @ t$, HighRotorSpeed $(x) @ t, \quad M a i n F l a m e O n(x) @ t$ and PurginglsOverFor1Tb $(x) @ t$. The first three queries are based on the definitions in Section 2, Regarding the query: PurginglsOverFor1Tb(tb0)@x, since the available data contains measurements only for one turbine, we define it by simplifying PurginglsOver as follows:

$$
\begin{aligned}
& \text { PurginglsOverFor1Tb }(t b) \leftarrow \text { MainFlameOn }(t b), \\
& \diamond_{(0,10 m]}\left(\Theta_{(0,30 s]} \text { HighRotorSpeed }(t b),\right. \\
& \left.\diamond_{(0,2 m]} \boxminus_{(0,1 m]} \text { LowRotorSpeed }(t b)\right) .
\end{aligned}
$$

The running times of these queries are shown in Fig. 2. As can be seen, they scale linearly. In particular the running times of PurginglsOverFor $1 \mathrm{~Tb}(\mathrm{tb} 0) @ x$, which contains all the other queries as subcomponents, provide an indication that our algorithm respects modularity.

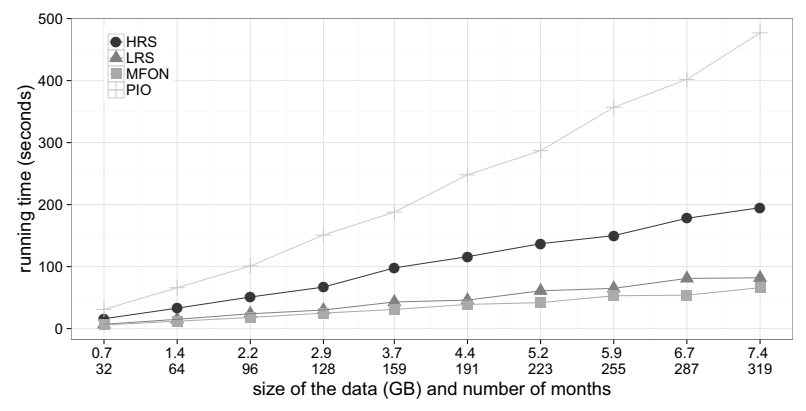

Fig. 2. Experiments with Siemens data over 32-319 months.

\section{Related work}

In this paper, our ontology language uses the operators of the metric temporal logic MTL that was originally introduced for modeling and reasoning about real-time systems (Koymans, 1990; Alur and Henzinger, 1993). Initial experimental results obtained by Brandt et al. (2018) demonstrated reasonably good performance and scalability on data from weather stations in the USA (of a size up to $8 \mathrm{~GB}$ ) and turbine sensor data (of a size up to $6 \mathrm{~GB})$. Another application of a similar formalism has been provided by El Raheb et al. (2017) in the context of querying user annotated ballet movement videos.

Practical ontology-mediated query answering with temporal ontologies based on the Halpern-Shoham interval temporal logic, $\mathcal{H S}$ (Halpern and Shoham, 1991) was investigated by Kontchakov et al. (2016). We remind the reader that $\mathcal{H S}$ is a classical propositional logic enriched with modal diamond operators of the form $\langle R\rangle$, where $R$ is one of the twelve interval relations by Allen (1983): After, Begins, Ends, During, Later, Overlaps, and their inverses. One of the use-cases reported by Kontchakov et al. (2016) deals with historical data about the Italian public administration, and the other one with the weather data mentioned above. The proposed implementation, based on a reduction to standard datalog reasoning (with arithmetic constraints), showed feasibility of the approach with several state-of-the-art datalog engines. We note that, in the papers discussed above, datalog is used as a static ontology language and conjunctive queries are used as a query language.

Temporal relational databases have been studied intensively since the 1990s. Notably, the TSQL2 query language was proposed by Snodgrass (1995) as a temporal extension of SQL92. Zimányi (2006) investigated temporal aggregations in temporal databases. Dignös et al. (2016) proposed a framework to implement temporal operators in a DBMS engine by extending its kernel. In this work, we do not assume that the underlying database supports additional temporal query language features (as those provided by TSQL2), and instead maintain compatibility with standard relational 
data sources. How to exploit in temporal OBDA additional features provided by temporal database query languages, when the underlying database supports them, is an interesting subject for future work.

\section{Conclusions}

In this paper, we have proposed a framework for practical temporal OBDA, defined its main components, and given a high level view of the system architecture.

As future work, we plan to formally present the working mechanism of the framework in detail, implement it as an extension of Ontop, and evaluate the implementation over the large scale heterogeneous Siemens use case data. Another future direction is to extend the framework in order to support semantic query answering over streaming data. There is an extensive body of work on semantic query answering over RDF streaming data (Barbieri et al., 2010; Calbimonte et al., 2012; Phuoc et al., 2011; Anicic et al., 2011; Beck et al., 2015; Özçep et al., 2014). However, none of these works follows the OBDA approach, apart from STARQL (Özçep et al., 2014), where one can define complex temporal patterns only at the query level rather than the ontology level as in our proposal. We plan to investigate how to incorporate the streaming data setting into our temporal OBDA framework.

\section{Acknowledgment}

This research has been partially supported by the Open Access Publishing Fund of the Free University of Bozen-Bolzano, by the projects OnProm, OBATS, and QUADRO, funded respectively through the 2015, 2017, and 2018 calls issued by the Research Committee of the Free University of Bozen-Bolzano, and by the Euregio IPN12 project KAOS funded through European Region Tyrol-South Tyrol-Trentino (EGTC) under the first call for basic research projects. The work of M. Zakharyaschev was carried out at the National Research University Higher School of Economics and supported by the Russian Science Foundation under the grant 17-11-01294.

This article is an extended and revised version of a paper that was published in the proceedings of the Workshop on Novel Techniques for Integrating Big Data (BigNovelTI 2017), co-located with the 21st European Conference on Advances in Databases and Information Systems (ADBIS 2017) (Brandt et al., 2017b).

\section{References}

Abiteboul, S., Hull, R. and Vianu, V. (1995). Foundations of Databases, Addison Wesley Publ. Co., Boston, MA.

Allen, J.F. (1983). Maintaining knowledge about temporal intervals, Communications of the ACM 26(11): 832-843.
Alur, R. and Henzinger, T.A. (1993). Real-time logics: Complexity and expressiveness, Information and Computation 104(1): 35-77.

Anicic, D., Fodor, P., Rudolph, S. and Stojanovic, N. (2011). EP-SPARQL: A unified language for event processing and stream reasoning, Proceedings of the 20th International World Wide Web Conference (WWW), Hyderabad, India, pp. 635-644.

Artale, A., Kontchakov, R., Kovtunova, A., Ryzhikov, V., Wolter, F. and Zakharyaschev, M. (2015a). First-order rewritability of temporal ontology-mediated queries, Proceedings of the 24th International Joint Conference on Artificial Intelligence (IJCAI), Buenos Aires, Argentina, pp. 2706-2712.

Artale, A., Kontchakov, R., Ryzhikov, V. and Zakharyaschev, M. (2015b). Tractable interval temporal propositional and description logics, Proceedings of the 29th AAAI Conference on Artificial Intelligence (AAAI), Austin, TX, USA, pp. 1417-1423.

Artale, A., Kontchakov, R., Wolter, F. and Zakharyaschev, M. (2013). Temporal description logic for ontology-based data access, Proceedings of the 23rd International Joint Conference on Artificial Intelligence (IJCAI), Beijing, China, pp. 711-717.

Baader, F., Borgwardt, S., Koopmann, P., Ozaki, A. and Thost, V. (2017). Metric temporal description logics with interval-rigid names, in C. Dixon and M. Finger (Eds.), Proceedings of the 11th International Symposium on Frontiers of Combining Systems (FroCoS), Lecture Notes in Computer Science, Vol. 10483, Springer, Berlin/Heidelberg, pp. 60-76.

Baader, F., Borgwardt, S. and Lippmann, M. (2013). Temporalizing ontology-based data access, Proceedings of the 24th International Conference on Automated Deduction (CADE), Lake Placid, NY, USA, pp. 330-344.

Baader, F., Calvanese, D., McGuinness, D., Nardi, D. and Patel-Schneider, P.F. (Eds.) (2007). The Description Logic Handbook: Theory, Implementation and Applications, 2nd Edn., Cambridge University Press, Cambridge.

Barbieri, D.F., Braga, D., Ceri, S., Valle, E.D. and Grossniklaus, M. (2010). C-SPARQL: A continuous query language for RDF data streams, International Journal of Semantic Computing 4(1): 3-25.

Beck, H., Dao-Tran, M., Eiter, T. and Fink, M. (2015). LARS: A logic-based framework for analyzing reasoning over streams, Proceedings of the 29th AAAI Conference on Artificial Intelligence (AAAI), Austin, TX, USA, pp. 1431-1438.

Borgwardt, S., Lippmann, M. and Thost, V. (2013). Temporal query answering in the description logic DL-Lite, in P. Fontaine et al. (Eds.), Proceedings of the 9th International Symposium on Frontiers of Combining Systems (FroCoS), Lecture Notes in Computer Science, Vol. 8152, Springer, Berlin/Heidelberg, pp. 165-180.

Brandt, S., Güzel Kalayci, E., Kontchakov, R., Ryzhikov, V., Xiao, G. and Zakharyaschev, M. (2017a). Ontology-based 
data access with a Horn fragment of metric temporal logic, Proceedings of the 31st AAAI Conference on Artificial Intelligence (AAAI), San Francisco, CA, USA, pp. 1070-1076.

Brandt, S., Güzel Kalayci, E., Ryzhikov, V., Xiao, G. and Zakharyaschev, M. (2017b). A framework for temporal ontology-based data access: A proposal, in M. Kirikova et al. (Eds.), New Trends in Databases and Information Systems, Communications in Computer and Information Science, Vol. 767, Springer, Berlin/Heidelberg, pp. 161-173.

Brandt, S., Güzel Kalayci, E., Ryzhikov, V., Xiao, G. and Zakharyaschev, M. (2018). Querying log data with metric temporal logic, Journal of Artificial Intelligence Research 62: 829-877.

Calbimonte, J.-P., Jeung, H., Corcho, Ó. and Aberer, K. (2012). Enabling query technologies for the semantic sensor web, International Journal on Semantic Web and Information Systems 8(1): 43-63.

Calvanese, D., Cogrel, B., Komla-Ebri, S., Kontchakov, R., Lanti, D., Rezk, M., Rodriguez-Muro, M. and Xiao, G. (2017). Ontop: Answering SPARQL queries over relational databases, Semantic Web Journal 8(3): 471-487.

Calvanese, D. and De Giacomo, G. (2003). Expressive description logics, in F. Baader et al. (Eds.), The Description Logic Handbook: Theory, Implementation, and Applications, Cambridge University Press, Cambridge, pp. 178-218.

Calvanese, D., De Giacomo, G., Lembo, D., Lenzerini, M. and Rosati, R. (2007). Tractable reasoning and efficient query answering in description logics: The DL-Lite family, Journal of Automated Reasoning 39(3): 385-429.

Cox, S. and Little, C. (2017). Time ontology in OWL, W3C Recommendation, W3C, https: //www.w3.org/TR/ owl-time/.

Das, S., Sundara, S. and Cyganiak, R. (2012). R2RML: RDB to RDF mapping language, W3C Recommendation, W3C, http://www.w3.org/TR/r2rml/

Dignös, A., Böhlen, M.H., Gamper, J. and Jensen, C.S. (2016). Extending the kernel of a relational DBMS with comprehensive support for sequenced temporal queries, ACM Transactions on Database Systems 41(4): $26: 1-26: 46$

El Raheb, K., Mailis, T., Ryzhikov, V., Papapetrou, N. and Ioannidis, Y. E. (2017). BalOnSe: Temporal aspects of dance movement and its ontological representation, in $\mathrm{E}$. Blomqvist et al.(Eds.), Proceedings of the 14th Extended Semantic Web Conference (ESWC), Vol. 10250, Springer, Berlin/Heidelberg, pp. 49-64.

Grandi, F. (2010). T-SPARQL: A SQL2-like temporal query language for RDF, Local Proceedings of the 14th EastEuropean Conference on Advances in Databases and Information Systems (ADBIS), Novi Sad, Serbia, pp. 21-30.

Gutiérrez-Basulto, V., Jung, J.C. and Kontchakov, R. (2016a). Temporalized EL ontologies for accessing temporal data:
Complexity of atomic queries, Proceedings of the 25th International Joint Conference on Artificial Intelligence (IJCAI), New York, NY, USA

Gutiérrez-Basulto, V., Jung, J.C. and Ozaki, A. (2016b). On metric temporal description logics, in G.A. Kaminka et al. (Eds.), Proceedings of the 22nd European Conference on Artificial Intelligence (ECAI), Frontiers in Artificial Intelligence and Applications, Vol. 285, IOS Press, Amsterdam, pp. 837-845.

Gutiérrez-Basulto, V. and Klarman, S. (2012). Towards a unifying approach to representing and querying temporal data in description logics, in M. Krötzsch and U. Straccia (Eds.), Proceedings of the 6th International Conference on Web Reasoning and Rule Systems (RR), Lecture Notes in Computer Science, Vol. 7497 Springer, Berlin/Heidelberg, pp. 90-105.

Gutiérrez, C., Hurtado, C.A. and Vaisman, A.A. (2005) Temporal RDF, in A. Gómez Pérez and J. Euzenat (Eds.), Proceedings of the 2nd European Semantic Web Conference (ESWC), Lecture Notes in Computer Science, Vol. 3532, Springer, Berlin/ Heidelberg, pp. 93-107.

Halpern, J.Y. and Shoham, Y. (1991). A propositional modal logic of time intervals, Journal of the ACM 38(4): 935-962.

Kharlamov, E., Brandt, S., Jiménez-Ruiz, E., Kotidis, Y., Lamparter, S., Mailis, T., Neuenstadt, C., Özçep, Ö. L., Pinkel, C., Svingos, C., Zheleznyakov, D., Horrocks, I., Ioannidis, Y.E. and Möller, R. (2016). Ontology-based integration of streaming and static relational data with Optique, Proceedings of the 2016 International Conference on Management of Data (SIGMOD), San Francisco, CA, USA, pp. 2109-2112.

Kharlamov, E., Mailis, T., Mehdi, G., Neuenstadt, C., Özçep, Ö. L., Roshchin, M., Solomakhina, N., Soylu, A., Svingos, C., Brandt, S., Giese, M., Ioannidis, Y.E., Lamparter, S., Möller, R., Kotidis, Y. and Waaler, A. (2017). Semantic access to streaming and static data at Siemens, Journal of Web Semantics 44: 54-74.

Klarman, S. and Meyer, T. (2014). Querying temporal databases via OWL 2 QL, in R. Kontchakov and M.-L. Mugnier (Eds.), Proceedings of the 8th International Conference on Web Reasoning and Rule Systems, Lecture Notes in Computer Science, Vol. 8741, Springer, Berlin/Heidelberg, pp. 92-107.

Kontchakov, R., Pandolfo, L., Pulina, L., Ryzhikov, V. and Zakharyaschev, M. (2016). Temporal and spatial OBDA with many-dimensional Halpern-Shoham logic, Proceedings of the 25th International Joint Conference on Artificial Intelligence (IJCAI), New York, NY, USA, pp. 1160-1166.

Kontchakov, R., Rezk, M., Rodriguez-Muro, M., Xiao, G. and Zakharyaschev, M. (2014). Answering SPARQL queries over databases under OWL 2 QL entailment regime, in P. Mika et al. (Eds.), Proceedings of the 13th International Semantic Web Conference (ISWC), Lecture Notes in Computer Science, Vol. 8796, Springer, Berlin/Heidelberg, pp. 552-567.

Koymans, R. (1990). Specifying real-time properties with Metric Temporal Logic, Real-Time Systems 2(4): 255-299. 
Möller, R., Özçep, Ö. L., Neuenstadt, C., Zheleznyakov, D. and Kharlamov, E. (2013). A semantics for temporal and stream-based query answering in an OBDA context, $O p$ tique Project Deliverable Deliverable D5.1, FP7-318338.

Özçep, Ö.L., Möller, R. and Neuenstadt, C. (2014). A stream-temporal query language for ontology based data access, in C. Lutz and M. Thielscher (Eds.), Proceedings of the 37th Annual German Conference on AI (KI), Lecture Notes in Computer Science, Vol. 8736, Springer, Berlin/Heidelberg, pp. 183-194.

Phuoc, D.L., Dao-Tran, M., Parreira, J.X. and Hauswirth, M. (2011). A native and adaptive approach for unified processing of linked streams and linked data, Lecture Notes in Computer Science, in L. Aroyo et al. (Eds.), Proceedings of the 10th International Semantic Web Conference (ISWC), Springer, Berlin/Heidelberg, pp. 370-388.

Poggi, A., Lembo, D., Calvanese, D., De Giacomo, G., Lenzerini, M. and Rosati, R. (2008). Linking data to ontologies, Journal on Data Semantics 4900: 133-173.

Rodriguez-Muro, M., Kontchakov, R. and Zakharyaschev, M. (2013). Ontology-based data access: Ontop of databases, in $\mathrm{H}$. Alani et al. (Eds.), Proceedings of the 12th International Semantic Web Conference (ISWC), Lecture Notes in Computer Science, Vol. 8218, Springer, Berlin/Heidelberg, pp. 558-573.

Snodgrass, R.T. (Ed.) (1995). The TSQL2 Temporal Query Language, Kluwer, Tucson, AZ.

Tappolet, J. and Bernstein, A. (2009). Applied temporal RDF: Efficient temporal querying of RDF data with SPARQL, in L. Aroyo et al. (Eds.), ESWC 2009, Lecture Notes in Computer Science, Vol. 5554, Springer, Berlin/Heidelberg, pp. 308-322.

Xiao, G., Calvanese, D., Kontchakov, R., Lembo, D., Poggi, A., Rosati, R. and Zakharyaschev, M. (2018). Ontology-based data access: A survey, Proceedings of the 27th International Joint Conference on Artificial Intelligence (IJCAI), Stockholm, Sweden, pp. 5511-5519.

Xiao, G., Rezk, M., Rodriguez-Muro, M. and Calvanese, D. (2014). Rules and ontology based data access, in R. Kontchakov and M.-L. Mugnier (Eds.), Proceedings of the 8th International Conference on Web Reasoning and Rules Systems (RR), Lecture Notes in Computer Science, Vol. 8741, Springer, Berlin/Heidelberg, pp. 157-172.

Zimányi, E. (2006). Temporal aggregates and temporal universal quantification in standard SQL, SIGMOD Record 35(2): 16-21.

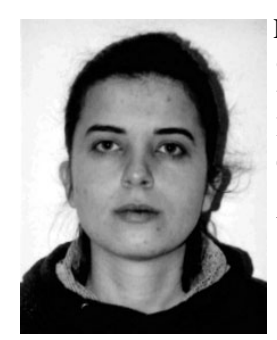

Elem Güzel Kalaycı is currently a PhD student of computer science at the KRDB Research Centre for Knowledge and Data, Free University of Bozen-Bolzano, Italy. Her main research interests are knowledge representation, temporal and stream reasoning, semantic web, and ontologybased data access.

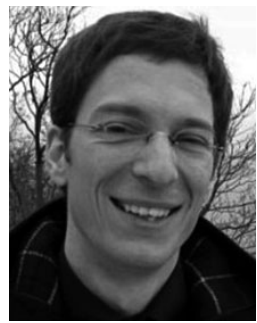

Sebastian Brandt received his $\mathrm{PhD}$ in computer science from the Technical University of Dresden, Germany, in 2006. He is a senior key expert at Siemens Corporate Technology. He is an expert in semantic technologies, reasoning, big data analytics, and knowledge representation formalisms, with the emphasis on lightweight ontology languages. His work has enabled the OWL 2 language profile OWL $2 \mathrm{EL}$, a W3C standard.

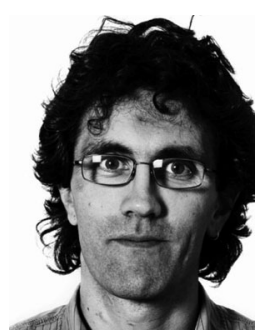

Diego Calvanese is a full professor at the KRDB Research Centre for Knowledge and Data, Free University of Bozen-Bolzano, Italy. His research interests include formalisms for knowledge representation and reasoning, ontology languages, description logics, conceptual data modeling, data integration, graph data management, data-aware process verification, and service modeling and synthesis. He is an editor of Description Logic Handbook. He has been a fellow of the European Association for Artificial Intelligence (EurAI) since 2015.

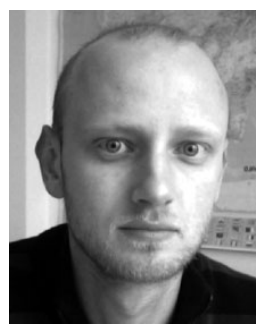

Vladislav Ryzhikov is a lecturer in computer science at Birkbeck, University of London. He had received his $\mathrm{BSc}$ and $\mathrm{MSc}$ degrees at Ufa Aviation State University, and was awarded a $\mathrm{PhD}$ in Computer Science at the Free University of Bozen-Bolzano, Italy, in 2010. His major research areas are artificial intelligence and knowledge representation. He has worked in a number of projects related to ontology-based data access.

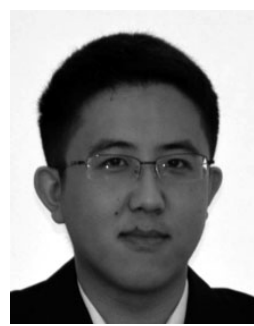

Guohui Xiao is an assistant professor at the KRDB Research Center for Knowledge and Data, Free University of Bozen-Bolzano, Italy. $\mathrm{He}$ received his $\mathrm{PhD}$ degree in computer science from the Vienna University of Technology, Austria, in 2014. He is currently leading the development within the Ontop team. His main research interests are knowledge representation, description logics, semantic web, database theory, ontology-based data access, and optimization and implementation of reasoning engines.

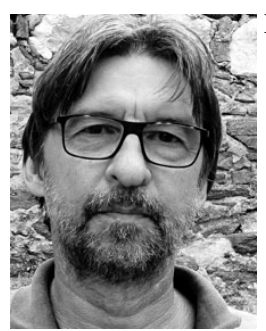

Michael Zakharyaschev is a professor of computer science at Birkbeck, University of London. He had received his BSc and MSc degrees at Moscow State University, and was then awarded a $\mathrm{PhD}$ and habilitation in mathematics at Novosibirsk State University. He has co-authored two major research monographs in modal logic. His current research areas are computational logic and its applications in knowledge representation, semantic technologies and, in particular, theory and practice of ontology-based data access. 


\section{Appendix \\ SQL functions (in plpgsql syntax) for temporal join and temporal interval coalesce}

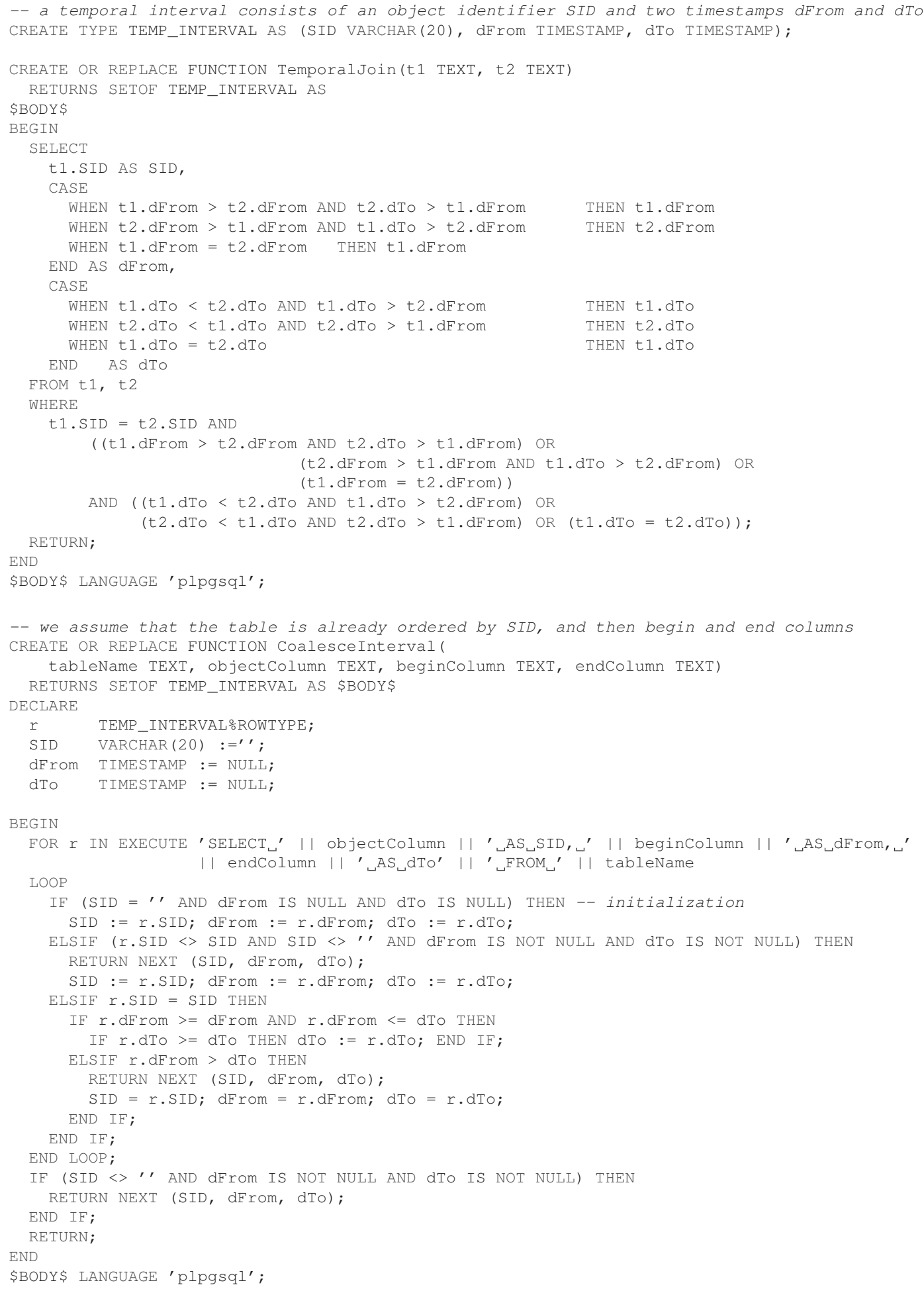

Received: 5 February 2018

Revised: 17 June 2018

Accepted: 22 September 2018 